\title{
Experiences and expectations of patients living with HIV on their engagement with care in Western Kenya
}

This article was published in the following Dove Press journal:

Patient Preference and Adherence

\author{
Juddy Wachira ${ }^{1,2}$ \\ Becky Genberg ${ }^{3}$ \\ Catherine $\mathrm{Kafu}^{2}$ \\ Paula Braitstein ${ }^{4}$ \\ Michael Barton Laws ${ }^{5}$ \\ Ira B Wilson ${ }^{5}$ \\ 'Department of Behavioral Sciences, \\ School of Medicine, Moi University, \\ Eldoret, Kenya; ${ }^{2}$ Department of \\ Epidemiology, Bloomberg School \\ of Public Health, John Hopkins \\ University, Baltimore, MD, USA; \\ ${ }^{3}$ Academic Model Providing Access to \\ Healthcare (AMPATH) Partnership, \\ Eldoret, Kenya; ${ }^{4}$ Dalla Lana School of \\ Public Health, University of Toronto, \\ Ontario, Canada; ${ }^{5}$ Department of \\ Health Services, Policy and Practice, \\ Brown University, Providence, RI, USA
}

Correspondence: Juddy Wachira Department of Behavioral Sciences, School of Medicine, Moi University, PO Box 4604-30100, Eldoret, Kenya Email wachirajuddy@gmail.com
Purpose: In resource-limited HIV care settings, effective and innovative interventions that respond to the existing challenges along the HIV care continuum are urgently needed to realize the benefits of antiretroviral therapy (ART). Initiating effective ART adherence dialog in an environment that promotes patient engagement in care is key. It is therefore critical to enhance our understanding about how patients living with HIV in these regions conceptualize and experience patient engagement. This study explores HIV patients' perceptions, experiences and expectations of their engagement in care.

Materials and methods: We sampled 86 patients from three Academic Model for Providing Access to Healthcare (AMPATHplus) sites, one urban and two rural. We conducted 24 in-depth interviews and eight focus group discussions in either Swahili or English. Audio recordings of the interviews were transcribed, and then translated into English. We performed content analysis after thematic coding.

Results: Patients living with HIV in Kenya desire active engagement with care. However, their engagement was inconsistent and varied depending on the provider. Patients had a sense of how provider's interpersonal behaviors influenced their level of engagement. These included various aspects of provider-patient communication and relationship dynamics. Patients also highlighted relational boundaries that influenced the level and kind of information they shared with their providers. Aspects of their psychological, social or economic wellbeing were often viewed as personal and not discussed with their clinicians. Patients identified factors that would promote or impede their engagement with care including those related to patients themselves, providers, and the healthcare system.

Conclusion: Patients living with HIV desired more active engagement in their care. In addition, they desired clinicians to engage in more social behaviors to promote patient engagement. To address existing patient engagement barriers, HIV care systems in the region should apply contextualized patient-centered interventions.

Keywords: patient engagement, HIV, Kenya

\section{Introduction}

Sub-Saharan Africa (SSA) continues to combat the spread and effects of HIV. ${ }^{1,2}$ Even though evidence-based prevention and treatment strategies have been implemented, SSA still bears a high burden of HIV compared with other regions. ${ }^{1-3}$ In the midst of the current "test and treat" guidelines where anyone testing HIV positive is immediately initiated on antiretroviral therapy (ART), ${ }^{4,5}$ efforts to keep patients fully engaged in care are necessary to ensure persistence with treatment. Patient engagement in care, the active involvement of patients in discussions and decisions regarding their care, ${ }^{6-8}$ has 
been shown to result in better ART adherence and improved clinical outcomes.

Barriers to patient engagement have been classified as intra-personal, inter-personal and system-level. ${ }^{9-13}$ Addressing system-level barriers is an important step in assisting patients to overcome their unique and varying intra and inter-personal challenges. At the system-level, high-income countries advocate for patient-centered care as the optimal approach to enhance patient engagement, ${ }^{14-16}$ and interventions in this setting usually focus on provider training. ${ }^{17}$ However, even in well-resourced settings, patient engagement can be difficult to achieve. SSA faces the challenges of poor health infrastructure, high patient volumes, limited systems to insure continuity of care, and a care system that tends to be physician-centered. ${ }^{18,19}$ Provider-focused patient engagement approaches that have been tested in high-income countries may not be applicable in SSA. Studies that explore how best to engage patients within a resource-constrained context, and that strive to understand local contextual factors, are urgently required to address the existing knowledge gap.

The overall goal of our study was to identify approaches to promote greater patient engagement in HIV care in western Kenya. The work described in this paper explores HIV patients' perceptions, experiences and expectations of their engagement in care. We used qualitative methods to address three main study questions: 1) How do patients living with HIV experience access to HIV care and ART adherence? 2) What are HIV patients' understanding and expectations of clinician-patient communication and patient engagement? 3) How do patients living with HIV individually experience clinician-patient communication and patient engagement?

\section{Material and methods}

\section{Study setting}

We conducted this study within the Academic Model for Providing Access to Healthcare (AMPATHplus) health facilities. AMPATHplus is a partnership between Moi Teaching and Referral Hospital (MTRH), Moi University, and a consortium of North-American Universities focused on delivering HIV care in western Kenya. ${ }^{20}$ We targeted one urban site (Moi Teaching and Referral Hospital, MTRH) and two rural sites (Webuye and Busia). MRTH is the largest urban AMPATHplus clinic representing a high ethnic and geographical diversity of patients living with HIV, located in Eldoret, Rift Valley Province. Webuye and Busia are rural sites where the majority of the residents are living below the poverty line.

AMPATHplus clinics are open every weekday between 8 am and 5 pm, but closed evenings and weekends. Clinics schedule return visits for new and non-adherent patients within 1-2 weeks in order to initiate the appropriate treatment in a timely way and to closely monitor those having difficulties with treatment adherence. Stable patients are given 3-6 months return dates. Clinic appointments refer to a day only, not to a time of day, and on the day that a patient has a return visit, patients are seen on a first come first serve basis. Patients see whichever provider is available when they reach the front of the line.

\section{Participants}

Eligible participants were: 1) 18 years and older, 2) confirmed to be HIV positive, 3 ) receiving HIV care at one of the three included AMPATHplus clinics, 4) on ART, and 5) willing to participate in the study. We excluded patients who were critically ill, unable to consent, or unable to speak in English or Swahili. Our protocol was reviewed and approved by both the Moi Teaching and Referral Hospital Institutional Research and Ethics Committee and the Institutional Review Board of Brown University. We obtained written consent from all participants. We conducted all interview sessions in private rooms and did not use participants' names during the interview sessions.

\section{Study design}

We conducted in-depth interviews and focus group discussions (FGD) between January and December of 2016. We recruited a purposive sample of patients until the predetermined total of 86 patients ( 24 in-depth interviews and 62 participants in eight FGDs) was achieved to fill the following strata: gender (female vs male), site (urban vs rural) and length in HIV care (newly enrolled $\geq 3$ months and $\leq 6$ months in HIV care vs older patients $\geq 12$ months in HIV care). We chose this number because based on prior experience, we believed this number would allow us to achieve saturation. ${ }^{21}$

\section{Conceptual framework for in-depth interviews and FGD}

The six function model of medical communication that links specific communication elements to concrete health outcomes ${ }^{22}$ was used to guide the development of the interview guides. This model includes the following constructs: 1) fostering the relationship, 2) gathering information, 3) information provision, 4) decision making, 5) enabling disease and treatment-related behavior, and 6) responding to emotions.

\section{Study procedures}

Each week, the clinical team in each of the three clinics identified eligible patients who would be attending clinic 
that week. Those interested in participating were referred to trained research assistants stationed in private rooms, who provided more information about the purpose of the study and obtained written consent.

We developed an interview guide with the following main domains: 1) patient socio-demographic characteristics of age, gender, education level, and ethnicity; 2) experiences with HIV testing and linkage to HIV care; 3) benefits and barriers to HIV care; 4) clinician-patient relationship expectations; 5) clinician-patient communication expectations; and 6) clinician-patient interaction experiences. We pretested the guide on a convenience sample of 8 patients from the AMPATH-MTRH clinic. Based on the pre-test findings, the guides were revised and translated to Swahili. Back translation was done to ensure that the Swahili and English versions were similar.

The eight FGDs each took 1.5 hours, and were done first, in order to inform the 24 in-depth interviews. In-depth interviews took approximately 45 minutes. We audio-recorded all sessions. We offered participants an inconvenience fee of 200 Kenyan Shillings (2-3 USD) at the end of the interview sessions. This amount is locally appropriate and is considered sufficient for lunch and transportation needs.

\section{Analyses}

We transcribed audio recordings verbatim, and the four interviews conducted in Swahili were translated to English. We used NVivo $8^{\mathrm{TM}}$ (QSR International) for coding and analysis. For validation, two investigators (JW and CK) conducted independent coding and identification of themes on twelve transcripts. These two investigators met regularly to discuss and agree on emerging and conflicting codes while revising the code book. Once the code book was finalized and consistency achieved, two additional trained research assistants assisted with coding. We used thematic analysis, ${ }^{23}$ with data coded under one or more themes. New themes were identified and added to pre-existing categories from the interview guide during the analysis. Data were scrutinized for differences and similarities within themes. The final results consisted of summaries, interpretations and textual excerpts.

\section{Results}

Of the 86 people approached, none declined to participate. Median age was 37 years, 48 (56\%) were females, and 79 $(93 \%)$ had at least a primary level of education.

We present findings according to five main themes that emerged from our analyses: 1) patients desired active engagement in care, 2) patients' experiences of engagement, 3) providers' interpersonal behaviors are critical for patient engagement, 4) rational boundaries, and 5) patient-identified factors that would facilitate their engagement.

\section{Patients desired active engagement in care}

All patients expressed a strong desire to actively participate in decisions regarding their care. The majority however felt that it was the clinician's responsibility to engage patients in treatment discussions and decision-making. This included clinicians taking a lead role in getting to know their patients' social and medical background. Patients defined engagement as the provider: 1) explaining to the patient the recommended treatment regimen, 2) considering the patient's opinion before taking any action, 3) asking for the patient's permission before ordering tests, and 4) discussing and agreeing before starting/changing a treatment regimen. They advocated for these actions as a way of making them feel more engaged.

He should ask me how the drugs he prescribed for me the last time have affected me or if I have experienced side effects. I will then inform that after taking those drugs I either had a headache or that drugs were okay, with no side effects. We can then progress from that point. [FGD 1 Male 12+ months]

When he wants to change the drugs for you, he should tell you the reasons. Not just writing the prescription in a hurry. I do not know if these pens have been cursed to have the clinicians write in a hurry. If you know the reasons you will not have a problem accepting those drugs because he has educated you. [FGD2 Male 12+ months]

You must discuss, he explains to you and you also give your opinion until you are content. It should not be that he just tells you. He should seek your opinion because it is important. [Male 12+ months]

\section{Patients' experience of engagement}

Despite their desire to actively participate in care, patients' experiences with providers varied. Those who had been in care for 12 months and more reported that they had experienced both clinicians who had engaged them as well as those who had not. Patients interacted with a number of clinicians during the course of their treatment.

We go to different rooms; you might go to a room (referring to clinician room) who will involve you. They are usually different because others just want to serve you and let you go. [FGD2 Male 12+ months]

On the other hand, patients who were newly enrolled in care (3-6 months in care) recognized the element of time as 
important in their engagement. Most of them felt that they had not yet been engaged, but were hopeful that it would happen with time.

They are yet to involve me but I am hopeful they will involve me [...] Time will tell. [Female 1-3 months]

\section{Clinicians' interpersonal behavior is critical for patient engagement}

A consistent emerging theme across all groups was the influence of providers' interpersonal behavior on patients' engagement with care. All patients desired that their clinicians make them feel welcomed, and noted both verbal and non-verbal ways in which this could be communicated. They reported to have experienced clinicians who were welcoming as well as those who were not welcoming.

Sometimes you get someone (clinician) who does not even greet you. But there are those who welcome you well. They even give you a seat and ask "So and so, how are you?" [...] "Do you have any problem? [...]" They just listen to you and that is nice because it means they have accepted your condition [...]. [FGD Female 3 months]

A majority of patients also appreciated clinicians who went beyond provision of care and showed concern about their socio-economic wellbeing.

I really like Dr X because every time you go there she must greet you, "How are you?" And ask "How are things at home? How do you live? What is the source of the water that you use? Is it from the well?" So you realize that this person is asking you all these questions for my own good. My wife told me that she has been told that if things get worse there is somewhere where we can get food. She told me that she has been told by the clinicians that if there is any problem for instance she needs flour for the baby's porridge she should go to a certain place [...]. [Male $12+$ months]

A few patients expressed the need to feel like a normal human being when they visited the health facilities. "Normal human beings" was an expression they used to refer to HIV negative individuals. They therefore expected clinicians to talk to them politely and respectfully.

[...] a relationship is the way they (providers) treat you when you get there (clinic), they treat you like a normal human being. Just like those who are not HIV positive. And when you ask them a question they answer you politely and nicely until you feel happy. You feel like the other people (HIV negative). [Male 12+ months]

\section{Patients expressed relational boundaries when engaging with their clinicians}

Although patients wanted physicians to be friendly and warm, they differed on the degree to which they wanted to share non-medical information with their clinicians. For some, issues not related to their physical wellbeing, including their psychological, social or economic wellbeing, were viewed as personal and not discussed with clinicians.

You could be having a problem at home such as not having food, you cannot tell him "give me some money to buy the children mandazi (snack) or food". There are some problems that you cannot say, you just keep quiet and struggle on your own. [FGD 4 Female 12+ months]

Some patients reported that they would only be open to their clinician, if the clinician asked them to.

If I have come to the clinic I will only respond to what he will ask of me. [Male 1-3 months]

When we got to the doctor, you enter and you are asked if you have a problem? [...] we respond according to what we have been asked although you may find that we have problems where we live. [FGD 2 Female 1-3 months]

Others stated that they would inform the clinician about their challenges with care only if they perceived that the clinician understood their situation. There were those who recalled instances where they feared telling their clinicians that they had missed their medication due to fear of being reprimanded.

It will only be easy if that clinician is someone whom when you talk to him he understands you, that way you will be free to talk to him without holding back anything. [FGD 1 Male 12+ months]

I was scared [...] I felt like I had done a mistake. You know there are some drugs like the ones for TB which you have to take daily $[\ldots]$ I feared he would get angry and shout at me so I just kept quiet. [Male 1-3 months]

Given the constant emphasis on adherence to treatment, a majority of patients expect clinicians not only to understand the challenges they are facing with care but also provide them with the necessary support. However, patients felt that some clinicians were not well informed about the treatment guidelines. They therefore did not see the need to share some of their concerns with such clinicians since they would not receive the right feedback.

At times when you talk to the clinician about the side effects he urges you to continue using those drugs and maybe 
because nowadays the drugs are combined they could be three drugs in one and maybe you are reacting to one of these three drugs. That is why I think some of the clinicians are yet to understand which drug in the combination is bringing about the side effects, or what is leading to this side effect, all they do is send you to the lab for viral load to check that it is suppressed but you as the patient you are suffering inside. [FGD 1 Male 12+ months]

\section{Patients identified factors that would facilitate their engagement}

Patients identified factors that made it easy or difficult for them to talk with their clinician, which included patient, provider and system-level factors. These factors were consistent among both newly enrolled patients and those who had been in care for 12 months and more.

Some patients felt obligated to talk with their clinicians regardless of whether the clinicians created a conducive environment.

Let me tell you, it is all about sacrifice especially now that I decided to voluntarily come here (referring to the HIV clinic)

$[\ldots]$ if there is a challenge that I am going through (referring to an ailment) it is a must I tell them. [Male 12+ months]

Despite this, patients emphasized the importance of provider-patient interaction time, the length of time patients have interacted with the care system, and support systems for patients. A few patients who had been in care more than 12 months expressed confidence in taking an active role in their care.

I think it depends on you because like for me I have been here for 10 years and I know that you should speak for yourself, it shouldn't be a situation like that of a police station where they are the only ones asking you questions. When you get to the clinician you should be knowing your condition because the time is limited you had better say what's ailing you so that you can be attended to quickly, if you go there and you do not speak up he will say "Do you want to be taken to your home in a casket because you are not using your drugs as prescribed" and you as a sick person you will not like such a talk [...] [FGD 1 Male 12+ months]

Patients with good adherence had more confidence about talking with their providers than those who had poor adherence. Some felt that physicians had a preference for adherent patients.

It becomes easy to talk to your clinician when you genuinely know that your adherence is good and that you are taking your drugs well but you are still falling ill. [FGD 1 Male

$12+$ months]

In addition, rapport between the patients and providers that leads to good provider-patient relationship created the right setting for dialog.

[...] It is usually easy for me to talk with them. There are those who are friends and when you meet them they want to talk to you and ask you how you are doing or the challenges you are facing and that shows that he cares about you and there is good relationship between you two. [Female 12+ months]

High patient volume was also seen as contributing to low patient engagement since clinicians were too fatigued to engage patients in their care.

Yes, it is not easy [...] It's not easy. I know for instance if the clinician has attended to like ten patients then you come in at $5 \mathrm{pm}$ and maybe he is tired he will not have time, the only time he will be having is time to write you a prescription so that you can go pick your drugs. He will not even ask you whether the drugs he prescribed to you the previous month affected you or not so that he can change for you the prescription. You cannot tell him that the drugs you prescribed for me the other week or month affected me maybe "I'm feeling weak, or I'm over sleeping or my body is really heating up" please change for the drugs. For him as long as you have enough drugs he doesn't have time to listen to what you have to say because there are many patients (waiting to be attended to). [FGD 1 Male 12+ months]

Finally, a few participants noted that peer support groups can be an avenue for patients to share experiences and be empowered about how to interact with clinicians and the care system.

For me it has been easy but it took time to get there, it is the sessions that I realized. [...] The group sessions that I used to attend. At times when used I go to the clinician and I didn't have money, I had struggled to even get fare to the clinic, getting food is a hustle, we did not think those were issues to let the clinician know so that he can give us relevant referrals. But nowadays I tell him that "honestly at times I forget to take my drugs on time" so that he can help me. [FGD 1 Male 12+ months]

\section{Discussion}

There are five main findings from this research. First, patients living with HIV in Kenya want higher levels of engagement with care. Second, levels of engagement are inconsistent, varying by provider. Third, providers' interpersonal skills 
influenced patients' level of engagement. Fourth, patients identified relational boundaries that influenced the kind of information they shared with their providers. Fifth, patients identified factors that would promote or impede their engagement with care including those related to the patients, providers, and health system. These findings provide insight into how patients view patient-centered care in western Kenya, and provide a foundation for developing contextualized patient-centered interventions within HIV care programs in SSA.

Even though there is evidence and support for patientcenteredness even in SSA, ${ }^{24,25}$ these are the first data that we are aware of that directly address how patients in SSA are currently thinking about patient-centeredness in HIV care. Studies have shown that patients generally have a wide range of preferences; some prefer that they or their providers make the most of the treatment decisions, while others desire shared decision making. ${ }^{26}$ Consistent with findings from other regions, ${ }^{27}$ our findings revealed that patients living with HIV generally want to take an "active role" in decisions about their HIV care. In a region that has historically had a paternalistic approach to care, ${ }^{28}$ these findings suggest that HIV care programs in the region may need to adopt a more patient-centered focus.

Despite patients' desire for more engagement with care, their engagement expectations were not always met. There have been similar finding in more developed contexts. ${ }^{26}$ A qualitative study conducted among HIV care providers in Kenya revealed that lack of adequate provider training and low system-level support were barriers to the adoption of patient-centered care. ${ }^{29}$ Patients in this region may therefore be ready to communicate freely with their clinicians about the challenges they face with their care, and to partner with their clinicians to identify solutions. However, providers, and the health systems in general, may not yet be well prepared for this level of engagement from their patients, in part due to resource constraints. Additional multilevel studies that apply more rigorous scientific approaches are needed to better understand these dynamics, as well as to identify suitable context-specific, patient-centered interventions.

Patients expressed that it is difficult to openly discuss their treatment views and concerns without the assent of clinicians. ${ }^{30}$ Patients clearly perceived that poor adherence negatively influenced clinicians' views of patients' engagement with care. ${ }^{29}$ Patients also clearly expressed the desire that clinicians provide higher quality interpersonal care, such as being more welcoming, concerned with patients' social wellbeing, and empathetic when patients experienced challenges with care. Providers who have a higher respect for their patients tend to engage in more rapport-building, social chitchat, and positive talk with their patients, thus promoting more patient-centeredness. ${ }^{31}$ Interventions that promote mutual respect between providers and patients and facilitate more substantive and meaningful provider-patient interaction are needed. Despite generally wanting clinicians to be respectful, friendly and warm, respondents differed in the degree to which they wanted to share personal and psycho-social information. ${ }^{32}$

Power imbalances between clinicians and patients are a major barrier to patient-centered care and shared decision making, ${ }^{33}$ and both clinicians and patients would benefit from training and skills development in these areas. For patients, this could involve taking advantage of peer support programs that have been shown to improve uptake of HIV care among patients. ${ }^{34}$ For clinicians, on-the-job training on provider-patient adherence communication ${ }^{35,36}$ as well as task shifting ${ }^{18}$ may be crucial to ensure that clinicians have the skill and time to adequately respond to the patients' needs. Emphasis should especially be placed on the value of clinicians interacting and supporting patients who may be viewed as non-adherent.

Health care systems in SSA continue to face a myriad of challenges, including high patient volume, low providerpatient ratio, and d clack of continuity of provider-patient relationship, that impede efforts to promote better engagement with care for patients living with HIV. ${ }^{18,19,24,37}$ In this context, the following three distinct but interacting layers have been identified as important: 1) factors that influence and shape the performance of providers; 2) structural and organizational features of the health system; and 3) the broader socioeconomic environment in which health workers operate. ${ }^{24}$ Our findings, through the eyes of patients, support the need for interventions on all three of these levels.

Our study has several limitations. First, AMPATHplus is a comprehensive HIV care program that is over 20 years old, and our findings may not generalize to settings with less well-established HIV care infrastructure. Second, while we suspect that our findings are generalizable to the wider Kenyan population, studies in other settings, both in Kenya and elsewhere in SSA, are needed.

\section{Conclusion}

In conclusion, we found that patients in AMPATHplus are anxious to be more involved with decision-making about their HIV care and would like their clinicians to be more supportive of that goal. Interventions that encourage and 
facilitate shared-decision making in HIV care in SSA are needed, and, at least in the setting that we studied, patients appear more ready for such interventions than clinicians. In addition to these clinician-patient communication challenges, patients identified a number of problems with HIV care that are more structural, and that will require different types of interventions.

\section{Acknowledgments}

The authors would like to thank all the study participants for taking part in the study. We would also like to acknowledge the data entry personnel, data managers, administrative and clerical staff, for enabling the management, interpretation, and publication of these data. We are also grateful to the AMPATH clinical and administrative staff, for their dedication in caring for patients, and their attentiveness in accurately recording their patients' data. This research was supported in part by a grant to the AMPATH Partnership from the United States Agency for International Development as part of the President's Emergency Plan for AIDS Relief (PEPFAR) and Lifespan/Tufts/Brown Center for AIDS Research (LTB CFAR NIH/NIAID fund P30AI042853). The contents of this study are the sole responsibility of AMPATH and do not necessarily reflect the views of USAID or the United States Government.

\section{Author contributions}

All authors made substantial contribution to the conceptualization and design, acquisition of data, analysis and interpretation of data. The first author (JW) drafted the article and all authors listed played an important role in revising the manuscript for intellectual content. All authors provided final approval of the version to be published and are accountable for all aspects of the work.

\section{Disclosure}

The authors report no conflicts of interest in this work.

\section{References}

1. Kharsany AB, Karim QA. HIV infection and AIDS in Sub-Saharan Africa: current status, challenges and opportunities. Open AIDS J. 2016;10:34-48.

2. Maartens G, Celum C, Lewin SR. HIV infection: epidemiology, pathogenesis, treatment, and prevention. Lancet. 2014;384(9939):258-271.

3. Abu-Raddad LJ, Barnabas RV, Janes H, et al. Have the explosive HIV epidemics in sub-Saharan Africa been driven by higher community viral load? AIDS. 2013;27(6):981-989.

4. Gardner EM, Mclees MP, Steiner JF, del Rio C, Burman WJ. The spectrum of engagement in HIV care and its relevance to test-and-treat strategies for prevention of HIV infection. Clin Infect Dis. 2011;52(6): 793-800.
5. Ministry of Health NA \& SCP. Guidelines on Use of antiretroviral drugs for treating and preventing HIV infection in Kenya. Nairobi, Kenya; 2016. Available from: https://www.prepwatch.org/wp-content/ uploads/2016/08/Guidelines-on-ARV-for-Treating-Preventing-HIVInfections-in-Kenya.pdf

6. Dang BN, Westbrook RA, Black WC, Rodriguez-Barradas MC, Giordano TP. Examining the link between patient satisfaction and adherence to HIV care: a structural equation model. PLoS One. 2013;8(1):e54729.

7. Rathert C, Wyrwich MD, Boren SA. Patient-centered care and outcomes: a systematic review of the literature. Med Care Res Rev. 2013; 70(4):351-379.

8. Aurelie Lucette GI. Doctor-patient relationship: active patient involvement (DPR:API) is related to long survival status and predicts adherence change in HIV. J AIDS Clin Res. 2015;6(2):427.

9. Barry MJ, Edgman-Levitan S. Shared decision making - pinnacle of patient-centered care. $N$ Engl J Med. 2012;366(9):780-781.

10. Bärnighausen T, Chaiyachati K, Chimbindi N, Peoples A, Haberer J, Newell ML. Interventions to increase antiretroviral adherence in subSaharan Africa: a systematic review of evaluation studies. Lancet Infect Dis. 2011;11(12):942-951.

11. Colvin CJ, Konopka S, Chalker JC, et al. A systematic review of health system barriers and enablers for antiretroviral therapy (ART) for HIV-infected pregnant and postpartum women. PLoS One. 2014;9(10):e108150.

12. Posse M, Meheus F, van Asten H, van der Ven A, Baltussen R. Barriers to access to antiretroviral treatment in developing countries: a review. Trop Med Int Health. 2008;13(7):904-913.

13. Hodgson I, Plummer ML, Konopka SN, et al. A systematic review of individual and contextual factors affecting ART initiation, adherence, and retention for HIV-infected pregnant and postpartum women. PLoS One. 2014;9(11):e111421.

14. Wachira J, Naanyu V, Genberg B, et al. Health facility barriers to HIV linkage and retention in Western Kenya. BMC Health Serv Res. 2014; 14(1):646.

15. Rathert C, Wyrwich MD, Boren SA. Patient-centered care and outcomes: a systematic review of the literature. Med Care Res Rev. 2013;70(4):351-379.

16. Epstein RM, Street RL. The values and value of patient-centered care. Ann Fam Med. 2011;9(2):100-103.

17. Levinson W, Lesser CS, Epstein RM. Developing physician communication skills for patient-centered care. Health Aff. 2010;29(7): 1310-1318.

18. Wilson IB, Laws MB, Safren SA, et al. Provider-focused intervention increases adherence-related dialogue but does not improve antiretroviral therapy adherence in persons with HIV. J Acquir Immune Defic Syndr. 2010;53(3):338-347.

19. Callaghan M, Ford N, Schneider H. A systematic review of task-shifting for HIV treatment and care in Africa. Hum Resour Health. 2010; $8(1): 1-9$.

20. Hontelez JA, Chang AY, Ogbuoji O, de Vlas SJ, Bärnighausen T, Atun R. Changing HIV treatment eligibility under health system constraints in sub-Saharan Africa: investment needs, population health gains, and cost-effectiveness. AIDS. 2016;30(15):2341-2350.

21. Einterz RM, Kimaiyo S, Mengech HN, et al. Responding to the HIV pandemic: the power of an academic medical partnership. Acad Med. 2007;82(8):812-818.

22. de Haes H, Bensing J. Endpoints in medical communication research, proposing a framework of functions and outcomes. Patient Educ Couns. 2009;74(3):287-294.

23. Fereday J, Muir-Cochrane E. Demonstrating Rigor Using Thematic Analysis: A Hybrid Approach of Inductive and Deductive Coding and Theme Development. Int J Qual Methods. 2006;5(1):80-92.

24. de MJ, Mayega RW, Sarkar N, et al. Patient-centered care and people-centered health systems in sub-Saharan Africa: why So Little of something so badly needed? Int J Pers Cent Med. 2016;6(3): $162-173$. 
25. Mcmillan SS, Kendall E, Sav A, et al. Patient-centered approaches to health care: a systematic review of randomized controlled trials. Med Care Res Rev. 2013;70(6):567-596.

26. Fuller SM, Koester KA, Guinness RR, Steward WT. Patients' Perceptions and Experiences of Shared Decision-Making in Primary HIV Care Clinics. J Assoc Nurses AIDS Care. 2017;28(1):75-84.

27. Chewning B, Bylund CL, Shah B, Arora NK, Gueguen JA, Makoul G. Patient preferences for shared decisions: A systematic review. Patient Educ Couns. 2012;86(1):9-18.

28. Norman I. Blind Trust in the Care-Giver: Is Paternalism Essential to the Health-Seeking Behavior of Patients in Sub-Saharan Africa? Adv Appl Sociol. 2015;5(2):94-104.

29. Wachira J, Genberg B, Kafu C, et al. The perspective of HIV providers in western Kenya on provider-patient relationships. J Health Commun. 2018;6:1-6.

30. Mallinson RK, Rajabiun $\mathrm{S}$, Coleman $\mathrm{S}$. The provider role in client engagement in HIV care. AIDS Patient Care STDS. 2007;21(Suppl 1): S77-S84.

31. Flickinger TE, Saha S, Roter D, et al. Respecting patients is associated with more patient-centered communication behaviors in clinical encounters. Patient Educ Couns. 2016;99(2):250-255.

32. Brooks K, Laws MB, Wilson I. Allocating HIV treatment to the adherent: a qualitative study of patient perceptions of their HIV care in the Dominican Republic. J Health Care Poor Underserved. 2014;25(4): 1748-1762.
33. Joseph-Williams N, Elwyn G, Edwards A. Knowledge is not power for patients: a systematic review and thematic synthesis of patient-reported barriers and facilitators to shared decision making. Patient Educ Couns. 2014;94(3):291-309.

34. Genberg BL, Shangani S, Sabatino K, et al. Improving engagement in the HIV care cascade: a systematic review of interventions involving people living with HIV/AIDS as Peers. AIDS Behav. 2016;20(10):2452-2463.

35. Conn VS, Ruppar TM, Enriquez M, Cooper PS, Chan KC. Healthcare provider targeted interventions to improve medication adherence: systematic review and meta-analysis. Int J Clin Pract. 2015;69(8): 889-899.

36. Beach MC, Roter DL, Saha S, et al. Impact of a brief patient and provider intervention to improve the quality of communication about medication adherence among HIV patients. Patient Educ Couns. 2015;98(9):1078-1083.

37. Blacklock C, Gonçalves Bradley DC, Mickan S, et al. Impact of contextual factors on the effect of interventions to improve health worker performance in Sub-Saharan Africa: review of randomised clinical trials. PLoS One. 2016;11(1):e0145206.
Patient Preference and Adherence

\section{Publish your work in this journal}

Patient Preference and Adherence is an international, peer-reviewed, open access journal that focuses on the growing importance of patient preference and adherence throughout the therapeutic continuum. Patient satisfaction, acceptability, quality of life, compliance, persistence and their role in developing new therapeutic modalities and compounds to optimize

\section{Dovepress}

clinical outcomes for existing disease states are major areas of interest for the journal. This journal has been accepted for indexing on PubMed Central. The manuscript management system is completely online and includes a very quick and fair peer-review system, which is all easy to use. Visit http://www dovepress.com/testimonials.php to read real quotes from published authors. 